\title{
Responsabilidad social empresarial y construcción de la marca: una nueva mirada a las estrategias de gestión
}

\section{Corporate social responsibility and branding: a new view to the management strategies}

\author{
Jorge Luis Del Río-Cortina' \\ Diego Cardona -Arbeláez ${ }^{2}$ \\ Abel Guacarí-Villalba ${ }^{3}$
}

Recibido: abril 15 de 2017

Aceptado: junio 29 de 2017

\section{Resumen}

La Responsabilidad Social Empresarial, RSE, se ha estudiado principalmente desde una perspectiva de desempeño financiero; de aquí que haya poco desarrollo entorno a la relación que existe entre la RSE y el proceso de construcción de marca, denominado Branding. El branding es importante como estrategia para generar efectos positivos en las empresas. Este artículo realiza una aproximación a esta discusión, aportando elementos que permiten entender cómo desde La responsabilidad social empresarial se puede gestionar la marca de las empresas, de modo que se generen estrategias que impacten positivamente la organización a todo nivel. Los hallazgos denotan que una adecuada gestión de la RSE, combinada con la gestión correcta de la marca puede tener incidencia en el crecimiento y en la rentabilidad de las empresas.

Palabras clave: responsabilidad social empresarial, marca, construcción de la marca, estrategias de gestión.

\begin{abstract}
Corporate Social Responsibility, CSR, has been studied mainly from a financial performance perspective; hence there is little development around the relationship between CSR and the process of brand building, called Branding. Branding is important as a strategy to generate positive effects in companies. This article makes an approximation to this discussion, providing elements that allow us to understand how from Corporate Social Responsibility can be managed the brand of the companies, so that strategies are generated which positively impact the organization at all levels. The findings suggest that an adequate management of CSR, combined with the correct management of the brand can have an impact on the growth and profitability of companies.
\end{abstract}

Keywords: corporate social responsibility, brand, branding, management strategies. 


\section{Introducción}

El concepto de Responsabilidad Social Empresarial, RSE, se origina formalmente en los trabajos de Bowen (1953), donde el autor refiere a la RSE cómo: "aquellas obligaciones de los empresarios en las políticas, decisiones y acciones deseables según los objetivos de la sociedad" (Moreno, Uriarte, \& Topa, 2010).

En las décadas de los 60 y 70, las empresas en aras de aumentar indicadores de eficiencia, eficacia y productividad, entre otros, descuidaban los efectos sociales que traía perseguir un modelo de empresa basado en la maximización del beneficio (Fierro-Ulloa, 2013). Este modelo consideraba los factores de producción, a saber: tierra, trabajo y capital, como recursos que se debían combinar de una manera eficiente para producir una mayor utilidad (Pertuz-Peralta, \& Perez-Orozco, 2016).

La utilización indiscriminada de recursos por parte de las empresas producía efectos negativos, tanto a la sociedad como al medio ambiente, puesto que no existían políticas que controlaran y mitigaran los daños producidos por aquella supuesta eficiencia (Medina-Monterrosa, \& Carvajalino-Slaghekke, 2014). En este sentido, las empresas que realizaban y aún hoy en día realizan acciones que no son socialmente responsables, generan una percepción negativa entre sus grupos de interés. Las universidades también tienen un papel fundamental desde lo cognitivo con la RSE, así como en el impacto social que se asocia con la participación en el desarrollo humano sostenible de la comunidad y su medio ambiente (Navas-Ríos, \& Romero-González, 2016).

Si bien se ha estudiado principalmente la manera como la responsabilidad social empresarial afecta el Desempeño financiero de la empresa, en la reciente literatura de RSE se han realizado esfuerzos para estudiar el impacto que generan estas políticas en las percepciones de los grupos de interés, conocidos como stakeholders en inglés (Vergara-Arrieta, 2015). El presente artículo reali- za una aproximación a esta discusión, aportando elementos que permiten entender cómo desde La responsabilidad social empresarial se puede gestionar la marca de las empresas, de modo que se generen estrategias que impacten positivamente la organización a todo nivel.

El documento inicia presentando los fundamentos sobre RSE y branding, junto con las posibilidades que su interrelación pueden brindar a la organización. Los hallazgos denotan que una adecuada gestión de la RSE, combinada con la gestión correcta de la marca pueden tener incidencia en el crecimiento y en la rentabilidad de las empresas.

\section{Aproximación teórica}

\subsection{Responsabilidad Social Empresarial}

La RSE es un nuevo paradigma de la gestión en las empresas, que ayuda al fortalecimiento de la responsabilidad de la empresa en la sociedad, p.ej. en cuestiones sociales y ambientales (Vergara-Arrieta, \& Carbal-Herrera, 2014). Una definición general de RSE la expresan McWilliams y Siegel (2001), como: "las acciones que aparecen para promover algún bien social, más allá de los intereses de la empresa y lo que es requerido por la ley". Así, no todas las acciones sociales que realice la empresa se pueden considerar dentro de la RSE, sino solo las que vayan más allá de los intereses de la empresa, en este caso de sus actividades de valor. Así mismo, el respaldo por parte del estado en fomentar en las empresas acciones que sean socialmente responsables, es importante puesto que estimula, a partir de beneficios, sobre todo fiscales, a las empresas que realicen este tipo de acciones (Martínez-Bernal, 2013).

Al respecto, la Comisión de las Comunidades Europeas, CCE, (2001), con la publicación del "Libro Verde", define la RSE cómo: "un concepto con arreglo al cual las empresas deciden voluntariamente contribuir al logro de una sociedad mejor y un medio ambiente más limpio". En esta misma línea, Carneiro-Caneda (2003), define a la RSE cómo: “La inte- 
gración voluntaria, por parte de las empresas de las preocupaciones sociales y morales en sus operaciones comerciales y en las relaciones con sus interlocutores". En relación con estas definiciones, se destaca que la decisión de las organizaciones en ser más responsable socialmente es netamente voluntaria, y eso dependerá de qué tanto esta sea más o menos responsable.
Es posible clasificar la aplicación de la Responsabilidad Social Empresarial desde dos dimensiones; Externa e Interna. Respecto a la dimensión Externa esta relaciona a la empresa con el entorno, mientras que la dimensión interna se refiere a la empresa en sí misma. Las dimensiones de la RSE se representan en la figura 1.

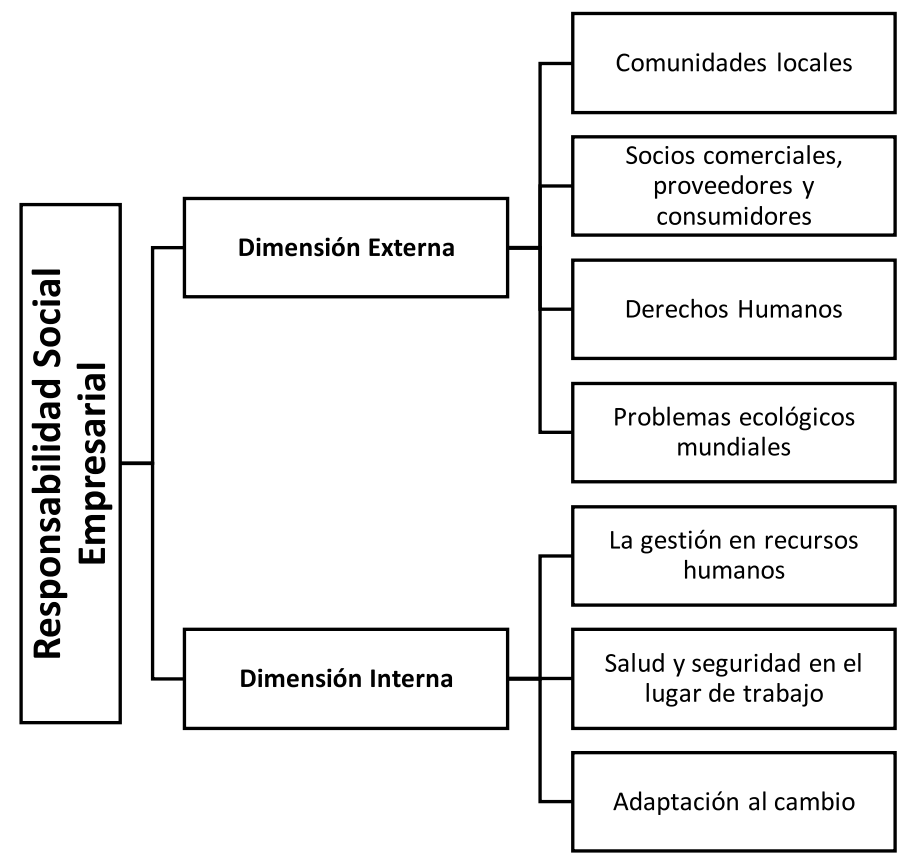

Figura 1. Dimensiones de la RSE, basadas en la CCE (2001).

\section{Dimensión externa}

En la dimensión externa de la RSE, de acuerdo con Carroll (1999), se pueden considerar:

Responsabilidad Social: se concreta en la realización de obras de interés social o cualquier otro tipo de práctica que suponga un beneficio social.

Responsabilidad Económica: la cual afecta a la búsqueda del máximo beneficio así como al logro del mayor valor posible para el accionista: ello se consigue a través de la mejora de la eficiencia y de la productividad.
Responsabilidad medioambiental: corresponde a la obligación que mantiene cualquier empresa de cuidar y preservar tanto el entorno como la naturaleza en su conjunto.

La CCE plantea otros elementos en cuanto a la dimensión externa de la RSE, los cuales se indican a continuación.

Comunidades locales. La RSE tiene en cuenta la integración de la empresa en el entorno donde se ubica. Es importante que la empresa contribuya al desarrollo de su entorno, teniendo en cuenta sobre todo el desarrollo de las comunidades locales; que se brinden mecanismos para la generación de empleos locales, generación de condiciones ópti- 
mas para la atención en salud y educación, además de servicios de agua potable y saneamiento básico. Es decir, todas las dimensiones que afectan al Índice de Desarrollo Humano, IDH (CCE, 2001).

Es muy importante el desarrollo de todos los aspectos de las comunidades locales, claro está que en concordancia con el desarrollo y crecimiento económico de la empresa (Pitre-Redondo, Cardona-Arbeláez, \& Hernández-Palma, 2017). En este sentido, es incongruente que una empresa se fortalezca cada año desde lo económico y su entorno esté deteriorado, sin acciones correctivas ni preventivas que puedan mejorar la problemática (Becerra-Gualdrón, \& Cruz-Vásquez, 2014). Así mismo, las empresas deben tener en cuenta el entorno físico local, el mantenimiento de un aire puro, aguas no contaminadas o carreteras descongestionadas tanto para su producción y oferta de servicios como para la calidad de vida de aquellas comunidades locales que conviven con la empresa (Alvarez-Meneses, 2013).

Socios comerciales, proveedores y consumidores. El beneficio de trabajar estrechamente con los socios comerciales de la empresa se traduce en la reducción de costos y en el fomento de la calidad del producto o servicio. Esta relación, a largo plazo, se puede traducir en precios, cláusulas y expectativas equitativas y suministros fiables y de calidad (CCE, 2001). De esta manera, las empresas deben ser conscientes de que la RSE de otra empresa socia puede afectar la práctica socialmente responsable de la empresa de diversas maneras.

En cuanto a ser socialmente responsables con los consumidores, se espera que las empresas ofrezcan de manera eficaz, ética y ecológica los productos o servicios hacia los consumidores, y que estos sean lo más transparentes en cuanto a su calidad y uso (Morales-Rubiano, Ortíz-Riaga, Duque-Orozco, \& Plata-Pacheco, 2017).

Derechos Humanos. En cuanto a los derechos humanos, la RSE está vinculada a actividades internacionales y las cadenas de suministros mundiales.
Las empresas multinacionales tienen en cuenta la Declaración tripartita de la Organización Internacional del Trabajo, OIT, y las directrices de la Organización para la Cooperación y el Desarrollo Econónico, OCDE, las cuales aplican en general para las empresas (Comisión de las Comunidades Europeas, 2001). La aplicación de códigos de conducta en cuanto a condiciones laborales, son adoptadas por la mayoría de las empresas, pues esto mejora su imagen empresarial y reduce el riesgo de que los consumidores reaccionen negativamente (Garrido-Hurtado, \& Valderrama-Cardona, 2016).

Problemas ecológicos mundiales. La importancia de que las empresas sean socialmente responsables con el medio ambiente, sobre todo con los problemas a escala internacional que afectan de manera global, hace que estas ejecuten políticas para un desarrollo sostenible, especialmente en materia de energía (Pereira-Blanco, 2015). Es frecuente la implementación de energías alternativas, que pueden mitigar el impacto medioambiental con respecto a las fuentes de energía no renovables, las cuales no son sostenibles ni sustentables a largo plazo (Reyes-Caballero, Fernández-Morales, \& Duarte, 2016).

\section{Dimensión interna}

La RSE interna trata sobre el talento humano dentro de la organización, en cómo ser socialmente responsables con los trabajadores. De acuerdo con Moreno y colaboradores (2010): "en la RSE interna la empresa debe incidir con especial atención en crear y fomentar una cultura de empresa, que esté presente de manera real y no fingida en todos sus miembros". Para lograr esta cultura, la empresa debe inculcar y fortalecer los valores en las personas, ya que finalmente son ellas las que hacen o denotan a la organización.

La responsabilidad social y el medio en que se mueve el trabajador deben ir más allá de los solos postulados en las políticas de la empresa; se necesita inversión de recursos económicos, conseguir un equilibrio entre el rol del trabajador y el papel 
que este representa en la sociedad (García-Rubiano, \& Forero-Aponte, 2015). Las empresas deben ver por encima de los muros y la productividad de sus empleados, deben visualizar los demás factores que pueden afectar el entorno de vida de sus funcionarios.

Así mismo, las empresas pueden encontrar en el fenómeno de la pobreza una oportunidad y convertirla en un negocio lucrativo, tal y como lo señalan Balza-Franco y Cardona-Arbeláez (2015): "Por su lado, las grandes compañías transnacionales -que en parte se benefician del fenómeno y también lo alimentan-, intentan mitigar los efectos de la pobreza con el desarrollo de diversos programas de RSE o con acciones de filantropía".

La CCE (2001), plantea que la dimensión interna de la RSE afecta a los trabajadores en aspectos cómo: la inversión en recursos humanos, la salud y la seguridad, y la gestión del cambio, como se indica a continuación:

La gestión en recursos humanos. El reto que afrontan las empresas en cuanto a los recursos humanos es en la atracción de trabajadores cualificados y en el mantenimiento de las personas en la organización (Parra-Penagos, \& Rodríguez-Fonseca, 2016). En este aspecto, la RSE debería tener: aprendizaje permanente, responsabilización de los trabajadores, mejora de información en la empresa, equilibro entre trabajo, familia y ocio, mayor diversidad de recursos humanos, igualdad de retribución y de perspectivas profesionales para las mujeres, entre otros. En últimas, la importancia de generar un entorno que estimule el aprendizaje permanente de los trabajadores, en particular de aquellos que tengan un menor nivel educativo, es uno de los mayores retos y apuestas que debe tener toda empresa que se considere en un ambiente de RSE (Comisión de las Comunidades Europeas, 2001).

Salud y seguridad en el lugar de trabajo. La normatividad sobre la salud y seguridad en el lugar de trabajo se ha venido fortaleciendo en los úl- timos años. El garantizar a los trabajadores condiciones óptimas para que puedan ejercer sus funciones dentro de la empresa es esencial, ya que afecta su eficiencia y productividad. Es decir, la calidad del proceso de producción repercute de manera directa en la calidad del producto o servicio que ofrece la empresa en el mercado, por lo tanto este es un tema crucial que impacta a las actividades de valor (CCE, 2001).

Ante esta situación, es importante que la empresa constantemente realice actualizaciones en sus planes de seguridad e higiene en el trabajo, debido a que estos afectan la calidad de la producción. Sin embargo, el aspecto de salud y seguridad en el trabajo no solo debe mirarse desde el punto de vista económico, sino que debe considerarse el factor humano, ya que la empresa debe garantizar la integridad del trabajador brindándole herramientas que minimicen los riesgos de un accidente laboral. Esto último debería ser la principal razón para la implementación de estos planes de seguridad.

Adaptación al cambio. La adaptación al cambio hace referencia a aquellos cambios en el entorno que afectan directamente a la empresa, ante los cuales se deben tomar decisiones internas que puedan adaptarla a las constantes dinámicas de los mercados (Novoa-Ruiz, 2013).

La CCE (2001), establece que aquella adaptación al cambio puede realizarse mediante una reestructuración de la empresa, pero que esta puede llevar consigo una reducción de los trabajadores. La reestructuración socialmente responsable, implica para la empresa el tener en cuenta los intereses y preocupaciones de los stakeholders afectados por el cambio. Además, la empresa debe asumir la responsabilidad en mantener la empleabilidad del personal, así como procurar proyectos que minimicen los efectos que pueda tener una reestructuración (Alean-Pico, del Río, Simancas-Trujillo, \& Rodríguez-Arias, 2017). 


\subsection{Branding}

\section{Antecedentes}

El branding es una disciplina que surgió a partir de la necesidad del dominio del consumo y especialmente de los bienes de gran consumo. Históricamente, ha sido relacionada con el producto (Gardner, \& Levy, 1955) y es visto como el proceso de creación de valor (Farquhar, 1989). Ésta disciplina, que para muchas personas es relativamente moderna, tiene una larga historia que incluso se remonta a mucho antes de la revolución industrial. Y aunque el concepto ha sido llevado a la práctica desde hace mucho tiempo, desde solo hace unos pocos años ha sido introducido y conceptualizado, atrayendo el interés de las organizaciones y de los académicos.

El temprano desarrollo de la escritura hizo crecer la necesidad de conversar acerca de dos cosas esencialmente: la religión y las transacciones de negocios (Walker, 1990). Para ayudar a estas operaciones transaccionales, los símbolos como forma de escribir eran utilizados con el objetivo de mantener un registro que iba ligado con las transacciones, y que decía cuanto estaba siendo transado (Moore, \& Reid, 2008). Es decir, que desde que las personas empezaron a hacer uso de las mercancías para intercambiar o vender ha habido marcas comerciales, carteles, símbolos y vendedores ambulantes, e incluso mucho antes, desde que las personas empezaron a marcar a los animales para denotar propiedad.

En ese orden de ideas, los artesanos para distinguir sus mercancías imprimían ciertas marcas en ellas, lo que las dirigía a un creador u origen, que en últimas se resumía en calidad para el comprador o cambista (Landa, 2005), catalogándose estos como los primeros indicios del logotipo, que es usado por Henderson y Cote (1998) para referirse al diseño gráfico que una compañía usa, con o sin su nombre para identificarse e identificar a sus productos.
Inicialmente el ganado era marcado con pintura y con alquitrán o brea de pino, y más tarde, desafortunadamente el ganado y las ovejas empezaron a ser marcados con hierros calientes. Los pueblos antiguos usaban las marcas para identificar la propiedad que tenían sobre ciertos bienes; la cerámica fue uno de los primeros productos que empezaron a marcarse, con la idea de dar cierta información acerca del dueño, los materiales utilizados y la época en que era creada, haciendo esto más específicamente cuando los productos eran vendidos o intercambiados (Pol, 2012). Los humanos también empezaron a ser marcados, y como evidencia de esto, tenemos a los esclavos y a los ladrones, los primeros eran marcados como símbolo de propiedad y los últimos como símbolo de repudio, desgracia y oprobio.

Aproximadamente en 1625, surge en Inglaterra el primer periódico de comunicación masiva. El comienzo del siglo XVIII marcó la aparición del conocido anuncio de periódico en los Estados Unidos, en el Boston Newslatter; que de ninguna manera parecido al de la actualidad, pues era muy limitado y contenía anuncios muy sencillos. Para atraer la atención de los lectores, muchos de los anuncios repetían varias líneas (Landa, 2005).

Con el desarrollo propiciado por la Revolución Industrial, las fábricas empezaron a usar la economía a escala, produciendo así una mayor cantidad de bienes que debían entonces ser vendidos a una mayor cantidad de personas, por lo que nace la necesidad de expandir los mercados. Rápidamente se dieron cuenta que el paquete genérico de un jabón tenía dificultades para competir con los ya familiarizados productos locales (Rajaram, \& Shelly, 2012). Es decir, se debían crear estrategias y diseñar paquetes que permitieran posicionar los productos, competir con las empresas locales, y estimular la demanda para alcanzar los mercados deseados. La producción en masa y la revolución industrial estimularon el crecimiento de la identificación visual y las marcas comerciales, lo que ilustró la importancia de las marcas comerciales y su identificación visual (Landa, 2005). Adicional- 
mente, el avance de tecnologías como: el teléfono, la fotografía, y el crecimiento de los medios de comunicación masivos, el mejoramiento de vías y la conexión entre pueblos, ciudades y países, el sistema postal, entre otros, no menos importantes, facilitaron el éxito de las marcas.

En el siglo XX, gracias al crecimiento industrial, muchos países, como es el caso de Estados Unidos en América, prosperaron. Muchas personas obtenían suficiente dinero para adquirir bienes extras, por lo que el mercadeo, la publicidad, el diseño gráfico y otras herramientas jugaron un papel importante para estimular la compra de bienes y servicios, contribuyendo así, al desarrollo y consolidación del concepto de marca, que en la actualidad sigue fortaleciéndose a través de los estudios científicos y académicos y de las experiencias empresariales.

\section{La gestión del Branding en las Empresas}

Las marcas, que hoy se encuentran por un sin número de lugares y que hacen parte del entorno, permiten crear juicios y prejuicios en torno a ellas, y se van construyendo a partir de las percepciones y los lazos emotivos que se logran establecer a través de la imagen, el lema, el producto, el servicio, entre otros factores que se conocen (Hernández-White, 2012), y que se logran o no percibir por medio de los sentidos. Lo anterior permite percibir la importancia del branding, pues se convierte en un proceso que ayuda a identificar, diferenciar y posicionar la marca en la mente del consumidor, trasmitiendo su esencia y expresando a los consumidores que la marca es única y exclusiva para ellos y que les genera unos beneficios.

En ese sentido, el branding es visto hoy como una herramienta de vital importancia en las organizaciones y se ha venido consolidando en los procesos de marketing, haciendo hincapié en la creación de marcas en sus actividades, a sabiendas de que en última instancia es en la mente del consumidor que reside la percepción de la marca (Kloter, \& Keller, 2012). Es por eso, que se ha hecho necesario para las organizaciones la utilización e implementación de esta poderosa estrategia.

La buena gestión del branding y la práctica del manejo de la marca son elementos clave de la estrategia de marketing (Keller, 2012) y debe ser de la competencia de todas las organizaciones, pues las afecta y tiene incidencia en el crecimiento y en la rentabilidad (Kapferer, 2008). Es decir, una buena gestión del branding permite resultados positivos y garantiza el aumento de las ventas, pues estos procesos afectan las preferencias de los consumidores e influencian en el proceso de toma de decisiones (Philiastides, \& Ratcliff, 2013), apelando a los sentidos para generar una experiencia positiva respecto a un producto o servicio (Gains, 2014), y entendiendo que no es solo a través de lo que se percibe por la vista, sino también de lo que se percibe por el tacto, el olfato, el gusto y el oído, que se crea identidad y un vínculo entre el consumidor y la marca (Lindström, 2005).

\section{RSE y branding: una aproximación práctica hacia una estrategia integradora}

La Responsabilidad Social Empresarial, RSE, afecta a la marca corporativa y a su gestión; a pesar de su importancia, este aspecto ha recibido poca atención por parte de los académicos. La literatura indica que la reputación de la empresa se ha estudiado desde diversas dimensiones, siendo el desempeño financiero el más desarrollado al respecto. Sin embargo, desde un punto de vista social y ambiental, existen muchos beneficios en los cuales la empresa debería gestionar la reputación de su marca (Attera, 2012). En este sentido, la RSE está íntimamente relacionada con las estrategias empresariales y puede permitir a las empresas la generación de valor, mediante la creación o adquisición de ventajas competitivas y mediante la mejora de la reputación de la corporación (Filho, Wanderley, Gómez, Farache, \& Francisca, 2010; Husted, \& Allen, 2000).

En el branding moderno la presencia de cualquier marca en el mercado no es tan importante como 
esa participación que tiene el producto en la mente del consumidor y que provoca la pregunta: ¿cuán importante es una marca específica para el consumidor? (Najdic, \& Bulovic, 2011). Esta pregunta lleva a las empresas a impulsar estrategias dentro de las organizaciones que permitan generar un concepto positivo de su marca en la mente del consumidor. Esto puede hacerse desde una perspectiva del marketing sustentable que genera oportunidades y ventajas competitivas, permitiendo que la empresa se diferencie de los competidores, generando un mejor posicionamiento de marca y que está ligada directamente con la RSE, entendiendo que cada vez los consumidores se sienten más comprometidos con el medio ambiente (Astaburuaga-Poblete, \& Kaltwasser-Bello, 2012).

Lo anterior hace que las empresas ofrezcan productos cada vez más amigables con el medio ambiente. Al respecto, Vilanova y colaboradores (2009), mencionan que las empresas terminan en cierta forma incorporando la RSE como parte de sus estrategias para mejorar la competitividad y su reputación, entendiendo que la competitividad de una firma es definida por el mercado mismo.

Lo anterior permite deducir que existen múltiples ventajas de utilizar la Responsabilidad social Empresarial asociada al branding. Entre las ventajas de emplear esa relación, se tienen: ayuda a construir la reputación de una marca, fomenta el interés del consumidor y ayuda a construir confianza y lealtad del consumidor. Si los consumidores ven que las marcas están abordando los temas que son importantes para ellos, es probable que continúen comprando sus productos (Verde-Nieto, 2009). Es a través de la RSE que las empresas pueden abordar directamente a los interesados, integrando a la comunidad y mejorando positivamente la reputación de la empresa (Attera, 2012; Melo, \& Galan, 2011).

La reputación y la legitimidad de la empresa pueden fortalecerse a través de la RSE, ya que es una manera práctica para que las empresas demuestren que se puede lograr la rentabilidad y al mismo tiempo satisfacer las demandas y necesidades de los interesados (Attera, 2012). Además, Cuando las empresas adoptan determinadas actividades de RSE, se puede construir relaciones sólidas con los grupos de interés, lo que redundará en importantes beneficios para las empresas en muchas áreas, como la lealtad del cliente y una baja rotación de personal.

En este contexto, las empresas no sólo deben lograr relaciones sólidas sino además generar un fuerte apoyo y el respeto de la comunidad. La creación y desarrollo empresarial debe ser integral ya que, si se aumenta el nivel de emprendimiento en una comunidad o región, esta presentará mejores indicadores de empleabilidad y disminución de pobreza, traduciéndose en mejor calidad de vida (Cardona-Arbeláez, Montenegro-Rada, \& Hernández-Palma, 2017).

En general, los beneficios de gestionar la reputación de la marca en una empresa, a través de la responsabilidad social empresarial, son (Attera, 2012): las personas desean trabajar en una compañía que tenga buena reputación, las empresas que son más responsables en sus contextos recibirán aplicantes con altas cualidades; además, Compañías que ofrecen igualdad de oportunidades a sus empleados pueden mejorar en el largo plazo el valor del accionista, reduciendo costos y riesgos.

Finalmente, las empresas que están involucradas con RSE, tienden a tener un alto porcentaje de empleados más creativos con un alto rendimiento, lo que conlleva a que los clientes sean más leales al percibir la responsabilidad social de la empresa. En este sentido, cuando los clientes perciben a la empresa como socialmente responsable, se ha conseguido su posicionamiento como una empresa socialmente responsable, lo cual se constituye en una de las ventajas competitivas más importantes. 


\section{Conclusiones}

La relación entre la Responsabilidad Social Empresarial, RSE, y el Branding ha recibido poca atención en la literatura especializada. No obstante, se ha empezado a gestionar la manera de cómo ser Socialmente Responsable generando una confianza en la reputación de la marca por parte de los grupos de interés de las empresas.

La buena gestión del branding y la práctica del manejo de la marca son elementos clave de la estrategia de marketing y debe ser de la competencia de todas las organizaciones, pues las afecta y tiene incidencia en su crecimiento y en la rentabilidad.

La reputación y la legitimidad de la empresa pueden fortalecerse a través de la RSE, ya que es una manera práctica, como señala Attera (2012), para que las empresas demuestren que se puede lograr la rentabilidad y al mismo tiempo satisfacer las demandas y necesidades de los interesados

\section{Referencias}

Alean-Pico, A., Del Rio, J., Simancas-Trujillo, R., \& Rodríguez-Arias, C. (2017). ¿El emprendimiento como estrategia para el desarrollo humano y social?. Saber, Ciencia Y Libertad, 12 (1). Recuperado de: http://www.sabercienciaylibertad.org/ojs/index.php/scyl/article/view/218

Alvarez-Meneses, T. (2013). La planificación turística: un aspecto clave para el desarrollo sostenible y regional de Boyacá. Revista de Investigación, Desarrollo e Innovación, 3 (2), 101-110. doi: http:// dx.doi.org/10.19053/20278306.2169

Astaburuaga-Poblete, P., \& Kaltwasser-Bello, F. (2012). Responsabilidad social empresarial y marketing sustentable: análisis cualitativo a nivel de empresas en Chile. Universidad de Chile.

Attera, M. M. (2012). Corporate reputation and its social responsibility: A comprehensive vision. Cua- dernos de Estudios Empresariales, 22, 129-149. doi: http://doi.org/10.5209/rev_CESE.2012.v22.44648

Balza-Franco, V., \& Cardona-Arbeláez, D. (2015). La responsabilidad social empresarial y la lucha contra la pobreza. Saber, Ciencia y Libertad, 10 (1), 115-124. doi: http://dx.doi.org/10.22525/sabcliber.2015v10n1.115124.

Becerra-Gualdrón, C. J., \& Cruz-Vásquez, J. L. (2014). Diagnóstico de la competitividad agroindustrial en el departamento de Boyacá para el año 2011. Revista de Investigación, Desarrollo e Innovación, 4 (2), 111-123. Doi: http://dx.doi. org/10.19053/20278306.2961

Bowen, H. R. (1953). Social responsibilities of the businessman. Harper.

Cardona-Arbeláez, D., Montenegro-Rada, A., \& Hernández-Palma, H. (2017). Creación de empresa como pilar para el desarrollo social e integral de la región caribe: Apuntes críticos. Saber, Ciencia y Libertad, 12 (1), 130-139. Recuperado de: http:// www.sabercienciaylibertad.org/ojs/index.php/ scyl/article/view/220.

Carneiro-Caneda, M. (2003). La responsabilidad social corporativa interna: la nueva frontera de los recursos humanos. Aedipe: Revista de la Asociación Española de Dirección de Personal, 27, 42-48.

Carroll, A. B. (1999). Corporate social responsibility: Evolution of a definitional construct. Business \& society, 38 (3), 268-295.

Comisión de las Comunidades Europeas, CCE. (2001). El libro verde: Fomentar un marco europeo para la responsabilidad social de las empresas, 1-35.

Farquhar, P. H. (1989). Managing Brand Equity. Marketing Research, 1 (3), 24-33. Recuperado de: http://doi.org/10.2307/1252048 
Fierro-Ulloa, I. (2013). Comportamiento organizacional positivo: implicaciones para la organización actual. Saber, Ciencia y Libertad, 8 (2), 103-111. Recuperado de: https://dialnet.unirioja.es/servlet/articulo?codigo $=5104984$

Filho, J. M. de S., Wanderley, L. S. O., Gómez, C. P., \& Farache, F. (2010). Strategic Corporate Social Responsibility Management for Competitive Advantage. BAR - Brazilian Administration Review, 7, (3), 294-309.

Gains, N. (2014). Brand essense. Using sense, symbol and story to design bran identity. London and Philadelphia: Kogan Page Limited.

García-Rubiano, M., \& Forero-Aponte, C. (2015). Contrato psicológico y cambio organizacional en una entidad perteneciente al sector terciario de la ciudad de Bogotá, Colombia. Revista de Investigación, Desarrollo e Innovación, 6 (1), 15-28. doi: http://doi.org/10.19053/20278306.4047

Gardner, B. B., \& Levy, S. J. (1955). The Product and the Brand. Harvard Business Review, 33 (2), 33-39. Recuperado de: http://doi.org/10.1016/07376782(88)90037-9

Garrido-Hurtado, D., \& Valderrama-Cardona, E. (2016). Identificación de las ideas de negocio para la creación de empresa en la población desplazada de Florencia, Colombia. Revista de Investigación, Desarrollo e Innovación, 7 (1), 3748. doi: https://doi.org/10.19053/20278306. v7.n1.2016.5634

Henderson, P. W., \& Cote, J. a. (1998). Guidelines for Selecting or Modifying Logos. Journal of Marketing, 62 (2), 14-30. http://doi. org/10.2307/1252158
Hernández-White, R. (2012). Branding sustentable. Revista del Centro de Investigación de la Universidad de la Salle, 10, (37) 95-99.

Husted, B. W., \& Allen, D. B. (2000). Is It Ethical to Use Ethics as Strategy?. Journal of Business Ethics, 27 (1), 21-31. http://doi. org/10.1023/A:1006422704548

Kapferer, J. (2008). The New Strategic Brand Management: Creating and Sustaining Brand Equity Long Term. London: Kogan-Page .

Keller, K. (2012). Strategic Brand Management: Building, Measuring, and Managing Brand Equity, 4th Edition. PEARSON.

Kloter, P., \& Keller, K. (2012). Dirección de Marketing. Journal of Chemical Information and Modeling (53). Pearson Educación.

Landa, R. (2005). Designing Brand Experience: Creating Powerful Integrated Brand Solutions. USA: Cengage Learning.

Lindström, M. (2005). Brand sense. How to Build Powerful Brands Through Touch, Taste, Smell, Sight and Sound. Kogan Page Limited.

Martínez-Bernal, M. S. (2013). Determinación de la productividad y competitividad de la pequeña minería del distrito minero del norte de Boyacá. Revista de Investigación, Desarrollo e Innovación, 3 (2), 72-86. doi: 10.19053/20278306.2168

McWilliams, A., \& Siegel, D. (2001). Corporate social responsibility: A theory of the firm perspective. Academy of management Review, 26 (1), 117-127. doi: http://doi.org/10.5465/ AMR.2001.4011987

Medina-Monterrosa, P., \& Carvajalino-Slaghekke, A. (2014). Ni al tigre ni al cuero: la toma de 
decisiones y el uso los sistemas de soporte y de ayuda a la decisión SSD-SAD. Saber, Ciencia y Libertad, 9 (1), 115-128. Recuperado de: http:// www.sabercienciaylibertad.com/ojs/index.php/ scyl/article/view/26

Melo, T., \& Galan, J. I. (2011). Effects of corporate social responsibility on brand value. Journal of Brand Management, 18 (6), 423-437. http://doi. org/10.1057/bm.2010.54

Moore, K., \& Reid, S. (2008). The birth of brand: 4000 years of branding. Business History, 50 (4), 419-432. Recuperado de: http://doi. org/10.1080/00076790802106299

Moreno, A., Uriarte, L. M., \& Topa, G. (2010). La responsabilidad social empresarial: Oportunidades estratégicas, organizativas y de recursos humanos. Ediciones Pirámide.

Najdic, M., \& Bulovic, V. (2011). 7th Global Brand Conference of the Academy of Marketing's Brand, Corporate Identity and Reputation SIG. En Emotional Branding in Tourism 4-7. Oxford.

Navas-Ríos, M., \& Romero-González, Z. (2016). Responsabilidad social universitaria, impactos de la Universidad Libre, sede Cartagena, en su gestión socialmente responsable. Saber, Ciencia Y Libertad, 11 (1), 187-196. doi: http://dx.doi. org/10.22525/sabcliber.2016v11n1.187196

Novoa-Ruiz, J. A. (2013). La cuestión integral del siglo XXI. Saber, Ciencia y Libertad, 8 (1), 71-83. Recuperado de: http://www.sabercienciaylibertad.com/ojs/index.php/scyl/article/view/116

Parra-Penagos, C., \& Rodríguez-Fonseca, F. (2016). La capacitación y su efecto en la calidad dentro de las organizaciones. Revista de Investigación, Desarrollo e Innovación, 6 (2), 131-146. doi: http://dx.doi.org/10.19053/20278306.4602
Pereira-Blanco, M. J. (2015). Relación entre energía, medio ambiente y desarrollo económico a partir del análisis jurídico de las energías renovables en Colombia. Saber, Ciencia y Libertad, 10 (1), 35-60. doi: http://dx.doi.org/10.22525/sabcliber.2015v10n1.3660

Pertuz-Peralta, V., \& Perez-Orozco, A. (2016). Modelo de cultura organizacional innovadora en caficultores del departamento del Cesar, Colombia. Revista de Investigación, Desarrollo e Innovación, 6 (2), 117-130. doi: https://doi. org/10.19053/20278306.3687

Philiastides, M. G., \& Ratcliff, R. (2013). Influence of branding on preference-based decision making. Psychological science, 24 (7), 1208-15. http:// doi.org/10.1177/0956797612470701

Pitre-Redondo, R., Cardona-Arbeláez, D., \& Hernández-Palma, H. (2017). Proyección del emprendimiento indígena como mecanismo de competitividad en el postconflicto colombiano. Revista de Investigación, Desarrollo e Innovación, 7 (2), 231-240. doi: https://doi. org/10.19053/20278306.v7.n2.2017.6068

Pol, A. (2012). La marca: un signo de identificación visual y auditivo sinérgico. Cuadernos del Centro de Estudios en Diseño y Comunicación. Ensayos, (42), 205-220. Recuperado de: http:// www.scielo.org.ar/scielo.php?script=sci_arttext\&pid=S1853-35232012000400015\&lng=es\&tl$\mathrm{ng}=\mathrm{es}$.

Rajaram, S., \& Shelly, S. (2012). History of Branding. International Journal Of Social Sciences \& Interdisciplinary Research, 1 (3), 100-104.

Verde-Nieto, D. (2009). Corporate Social Responsibility as part of your brand - a new approach to engaging customers. British Journal od Administrative Management, 28-30. 
Vergara-Arrieta, J. J. (2015). Los sistemas de medición del desempeño estratégico frente a la competitividad y sustentabilidad. Saber, Ciencia y Libertad, 10 (2), 17-26. Recuperado de: https://dialnet.unirioja.es/servlet/articulo?codigo $=5295019$

Vergara-Arrieta, J., \& Carbal-Herrera, A. (2014). Diseño de un sistema de gestión en responsabilidad social empresarial para pequeños hoteles en la ciudad de Cartagena. Saber, Ciencia y Libertad, 9 (2), 91-108. doi: http://dx.doi. org/10.22525/sabcliber.2014v9n2.91108
Vilanova, M., Lozano, J. M., \& Arenas, D. (2009). Exploring the Nature of the Relationship Between CSR and Competitiveness. Journal of Business Ethics, 87 (1), 57-69. doi: http://doi.org/10.1007/ s10551-008-9812-2

Walker, C. B. (1990). Cuneiform. In J.T. Hooker (Ed.), Reading the past: ancient writing from $\mathrm{Cu}$ neiform to the alphabet, 15-74. London: British Museum Press. 